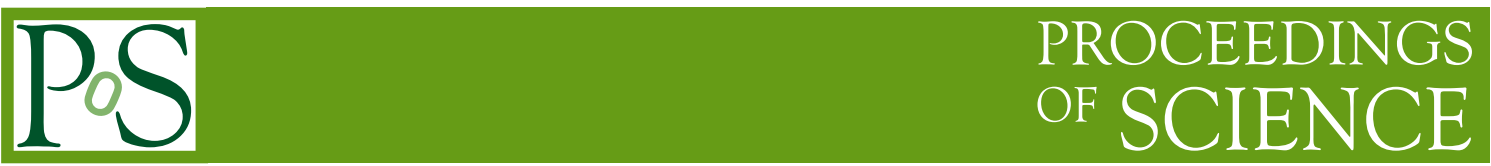

\title{
New physics searches with SHIP
}

\author{
Kang Young Lee* \\ Department of Physics Education \& RINS, Gyeongsang National University, Jinju 52828, Korea \\ E-mail: kylee.physegnu.ac.kr
}

\section{On behalf of SHiP collaboration}

\begin{abstract}
The SHiP Collaboration has proposed a general-purpose experimental facility operating in beam dump mode at the CERN SPS accelerator with the aim of searching for light, long-lived exotic particles of Hidden Sector models. The SHiP experiment incorporates a muon shield based on magnetic sweeping and two complementary apparatuses. The detector immediately downstream of the muon shield is optimized both for recoil signatures of light dark matter scattering and for tau neutrino physics. The second detector system aims at measuring the decays of hidden sector particles into the visible sector in a nearly background free environment. The detector consists of a $50 \mathrm{~m}$ long decay volume followed by a spectrometer and particle identification. Using the highintensity beam of $400 \mathrm{GeV}$ protons, the experiment is capable of integrating $2 \times 10^{20}$ protons in five years, which allows probing dark photons, dark scalars and pseudo-scalars, and heavy neutrinos with $\mathrm{GeV}$-scale masses at unprecedented sensitivities. The sensitivity to heavy neutrinos will allow for the first time to probe, in the mass range between the kaon and the charm meson mass, a coupling range for which baryogenesis and active neutrino masses can be explained. Following the review of the Technical Proposal, the CERN SPS Committee recommended in 2016 that the experiment and the beam dump facility studies proceed to a Comprehensive Design Study phase. These studies have resulted in a mature proposal submitted to the European Strategy for Particle Physics Update.
\end{abstract}

The 21st international workshop on neutrinos from accelerators (NuFact2019)

August 26 - August 31, 2019

Daegu, Korea

*Speaker. 


\section{Introduction}

Hidden sectors has drawn increasing attention as an alternative solution to problems which are not understood in the Standard Model (SM) framework, the dark matter, the origin of the baryon asymmetry, and the structure of neutrino masses etc.. Recently proposed is the new facility of the Search for Hidden Particles (SHiP) dedicated to searching for such kind of feebly interacting new particles $[1,2]$. The SHiP is a general purpose fixed target experiment using the CERN Super Proton Synchrotron (SPS) high intensity proton beams. The facility will accumulate $2 \times 10^{20}$ protons on target in five years to observe weakly interacting particles. The $400 \mathrm{GeV}$ proton beam impinging on the target could produce exotic particles between a few hundred $\mathrm{MeV}$ and a few GeV. Since such hidden particles are so long-lived that the decay lengths are of tens of meters, the facility possess very long volume more than hundred meters. The SHiP experiment will provide a promising intensity frontier research complimentary to the LHC at high energy frontier. The progress report is released in 2019 [3].

\section{The SHiP experiment}

The SHiP experiment will be located in the beam dump facility of the SPS at the North Area of the CERN. Figure 1 shows the configuration of the SHiP experiment. The SPS proton beam will be delivered onto the target which consists of blocks of titanium-zirconium doped molybdenum, followed by tungsten blocks. The main challenges of the SHiP concern the reduction of the SM background as efficiently as possible in order to maximize the sensitivity. An iron hadron stopper of five meters thickness after the target absorbs the produced hadron shower, and is followed by the 35 $\mathrm{m}$ long active muon shield which deflects muons with magnetic field to sweep away them from the detector acceptance. Downstream of the muon shield, the Scattering and Neutrino Detector (SND) is located, which aims at studying $\tau$ neutrinos and searching for the light dark matter directly. Please refer to Ref. [4] for the SND and the related physics.

The detector system for hidden sector consists of a $50 \mathrm{~m}$ long vacuum vessel for decay volume followed by a magnetic spectrometer with a rectangular acceptance of $5 \times 10 \mathrm{~m}^{2}$. The hidden sector particle is supposed to decay inside the vessel. The length of the decay volume is determined by

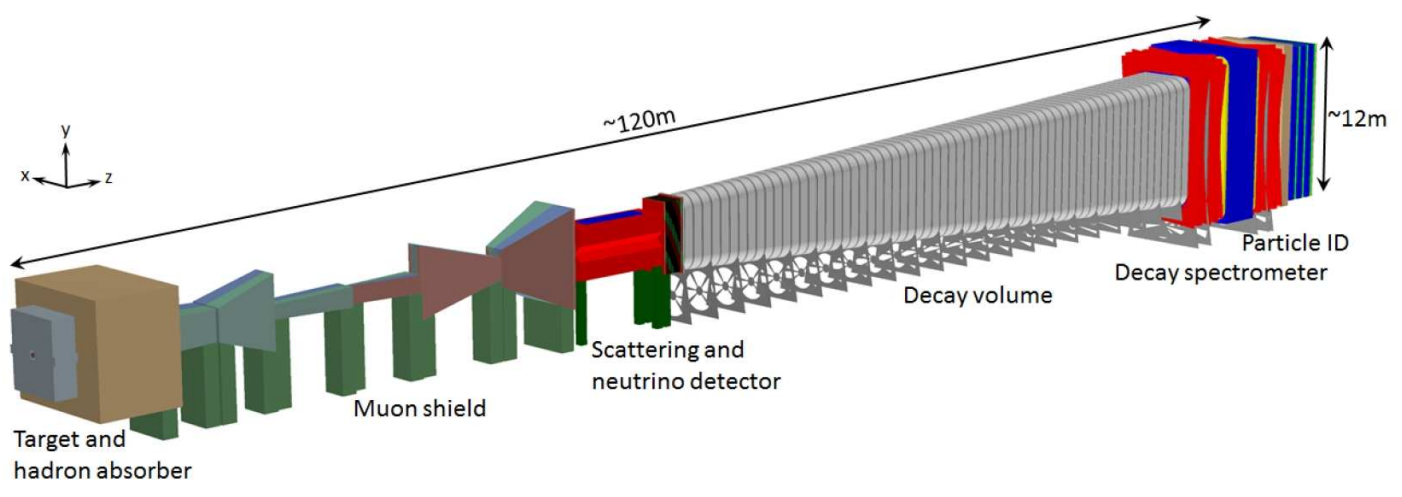

Figure 1: Overview of the SHiP experiment given in the SHiP CDS progress report [3] 


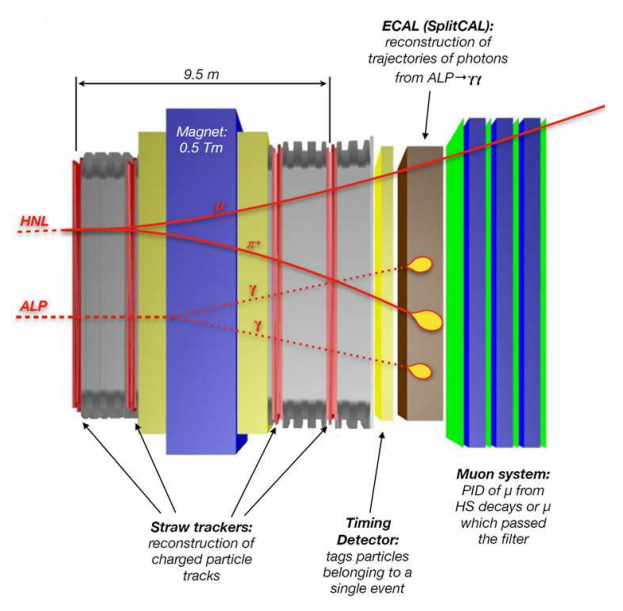

Figure 2: Layout of the hidden sector detector.

maximizing the acceptance to the hidden particle decay products given the transverse size of the spectrometer. The vessel is surrounded by a liquid scintillator layer to find the background particles from outside and maintained at a pressure below the mbar level to suppress a background induced by the inelastic neutrino interaction with the air in the fiducial volume. The spectrometer with straw trackers is designed to reconstruct the decay vertex, the mass and the trajectory of hidden sector with a high precision. Downstream of the spectrometer, a set of calorimeters and muon system provide particle identification, and a timing detector provides a measure of coincidence to reject combinatorial backgrounds. The layout of the designed detector is shown in Fig. 2.

\section{Searches for hidden sectors}

\subsection{Heavy Neutral Leptons}

The heavy neutral lepton (HNL) is the generic name of the new SM gauge singlet fermions, which couple to the left-handed lepton doublet through Yukawa interactions. Introducing the HNLs to the SM leads to one of the minimal extensions of the SM, so called the neutrino Minimal Standard Model, $v$ MSM, which have many virtues. The smallness of the neutrino masses is explained by the see-saw mechanism, the baryon asymmetry of the Universe is possible through the leptogenesis scenario, and the lightest HNL, if lighter than the electron, can be the dark matter candidate. In this case the other HNLs of GeV masses could be observed at the SHiP experiment [5]. The HNLs have the interactions with the SM particles through mixing with the active neutrinos. They will be produced from the weak decays of heavy hadrons and decays into charged leptons and hadrons. Recently the sensitivity of the SHiP experiment to the HNLs has been extensively studied in the Ref. [6].

\subsection{Dark Photons}

If the hidden sector contains $\mathrm{U}(1)_{X}$ gauge symmetry, it is possible that the field strength tensors of the $\mathrm{U}(1)_{X}$ and the $\mathrm{SMU}(1)_{B}$ are mixed in the kinetic terms. Then the $\mathrm{U}(1)_{X}$ gauge boson is mixed with the photon and coupled to the SM electromagnetic currents though suppressed by the small 

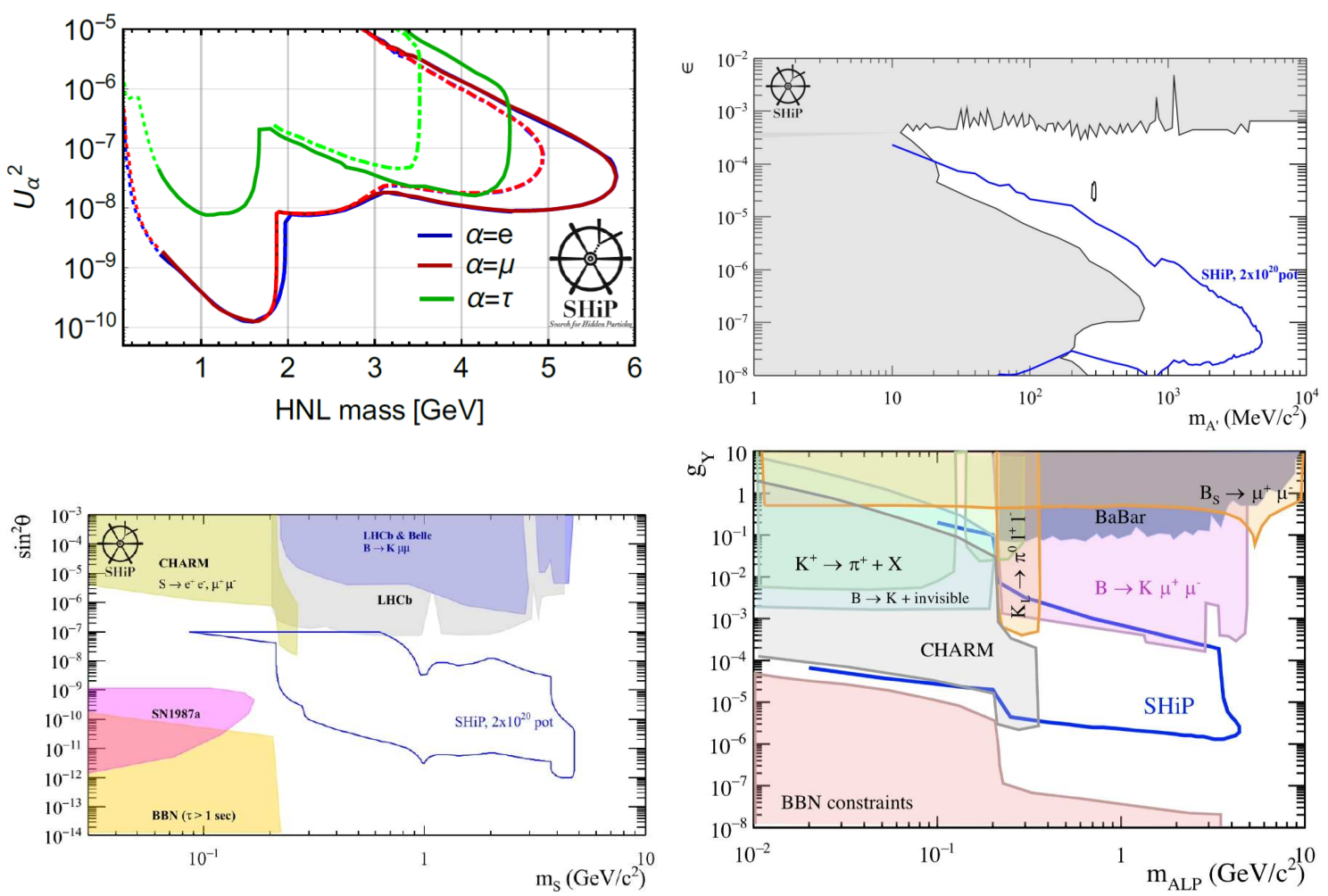

Figure 3: Left upper: SHiP sensitivity plot for HNLs mixing to a single SM flavour at $90 \%$ C.L. [6]. The solid curves assume the production fraction of $B_{c}$ meson same as that at the LHC energy and the dasheddotted curves do not include $B_{c}$ contributions. Right upper: Expected $90 \%$ exclusion region in the dark photon mass and the kinetic mixing parameter space [3]. Left lower: SHiP sensitivity to dark scalars [3]. Right lower: SHiP sensitivity to ALP coupling to fermions [3].

mixing angle. Hence the new gauge boson is referred to as the "dark photon" [7]. Since hidden sector particles can be connected to the SM particles through the dark photon mixing, we call this kinds of new physics models the vector portal. The dark photon may be produced from the meson decays, bremsstrahlung of proton, or the direct production of the QCD processes and decay into the lepton pair or hadron pair.

\subsection{Dark Scalars}

The quadratic term of the Higgs boson is the only term involving dimensionful coupling in the UV limit of the SM lagrangian. Thus hidden sectors with mass scales can have renormalizable couplings to the Higgs quadratic term. When those kinds of interactions are the only couplings between the hidden sector and the SM particles, we call the model the Higgs portal [8]. The simplest way is to couple a SM singlet scalar to the Higgs quadratic term through a triple and quartic couplings. After the spontaneous symmetry breaking, the singlet scalar is mixed with the Higgs boson and coupled to the SM particles via mixing. Such a dark scalar is produced from the heavy hadron decays and also sequently decays into hadrons and leptons in the vacuum vessel. 


\subsection{Axion Like Particles}

The axion is a very weakly interacting pseudoscalar Nambu-Goldstone boson of very small mass introduced to solve the strong $\mathrm{CP}$ problem. Such pseudoscalar particles may arise from spontaneous breaking of various symmetries at high energy scales. The SHiP experiment is not sensitive to the QCD axion which has been much studied to be highly constrained. Instead the SHiP searches for other pseudoscalar particles, very weakly interacting similarly to the axion but generically larger masses. We call this kind of particles the Axion-like particle (ALP) [9]. The ALPs couples to other particles via dimension-5 operators, e. g. derivative couplings to the axial vector current $\left(\partial_{\mu} a / f\right) \bar{\psi} \gamma^{\mu} \gamma^{5} \psi$ and coupling to the CP-odd kinetic terms of gauge fields $(a / f) F_{\mu \nu} \tilde{F}^{\mu v}$ etc.. As you see, the coupling is suppressed by the symmetry breaking scale $f$.

\section{Prospects}

The beam tests to measure the global performance of all of SHiP's subsystems are prepared in 2021. Final prototypes of the detector and the Technical Design Reports are scheduled to be prepared by end of 2022. The detector production is estimated to require two to three years and the detector assembly and installation will require another two years. The final installation of the facility and the detector is planned for the Long Shutdown 3 of the LHC and the data taking will start in LHC Run 4 period.

\section{Acknowledgments}

This work was supported by Basic Science Research Program through the NRF funded by the Ministry of Education under the Grant No. NRF-2018R1D1A3B07050649.

\section{References}

[1] S. Alekhin et al., SHiP collaboration, A facility to Search for Hidden Particles at the CERN SPS: the SHiP physics case, Rept. Prog. Phys. 79, 124201 (2016) [arXiv:1504.04855].

[2] M. Anelli et al., SHiP collaboration, A facility to Search for Hidden Particles (SHiP) at the CERN SPS, [arXiv:1504.04956].

[3] SHiP collaboration, SHiP Experiment, Progress Report, CERN-SPSC-2019-010/SPSC-SR-248.

[4] C. S. Yoon, Neutrino physics with the SHiP experiment at CERN, in this proceeding.

[5] T. Asaka, S. Blanchet, and M. Shaposhnikov, The vMSM, dark matter and neutrino masses, Phys. Lett. B 631, 151 (2005), [arXiv:hep-ph/0503065];

T. Asaka and M. Shaposhnikov, The vMSM, dark matter and baryon asymmetry of the universe, Phys. Lett. B 620, 17 (2005), [arXiv:hep-ph/0505013].

[6] C. Ahdida et al., SHiP collaboration, Sensitivity of the SHiP experiment to Heavy Neutral Leptons, JHEP 1904, 077 (2019), [arXiv:1811.00930].

[7] e.g. B. Holdom, Two U(1)'s and Epsilon Charge Shift, Phys. Lett. B 166, 196 (1986).

[8] B. Patt and F. Wilczek, arXiv:hep-ph/0605188.

[9] B. Doebrich, J. Jaeckel, F. Kahlhoefer, A. Ringwald, K. Schmidt-Hoberg, ALPtraum: ALP production in proton beam dump experiments JHEP 1602, 018 (2016), [arXiv:1512.0306]. 